\title{
Fabrication of Rare Earth Copolymerized Monodisperse Poly (Methyl Methacrylate) Microspheres Material
}

\author{
Changhao Yan, Yun Li, Huan Wang, Ming Zhang * \\ College of chemistry and chemical engineering \\ Yangzhou University \\ Yangzhou, China \\ e-mail: lxyzhangming@yzu.edu.cn
}

\begin{abstract}
A kind of rare earth copolymerizd monodisperse poly (methyl methacrylate) microspheres material was synthesized through dispersion polymerization. The chemical structure and chemical properties of the material were characterized by means of canning electronic microscope morphology (SEM), ultraviolet and visible spectrum (UV), dynamic laser scattering apparatus (DLS) and the thermogravimetric analysis (DTG). At the same time, the effects of different contents of monomer and rare earth content have already been investigated. SEM confirmed that the rare earth complexes have copolymerized. Meanwhile, with the content of rare earth, the microspheres show the characteristics absorption of rare earth, which will broaden the scope of application of microspheres.
\end{abstract}

Keywords-PMMA; microspheres; rare earth

\section{INTRODUCTION}

Since Vanderhoff's group firstly announced their preparation of polystyrene particles with highly uniform particles size in 1995, monodisperse polymer particles have found a wide variety of application [1-3] in coating, inks, dry toners, instrument calibration, chromatography, and biomedical treatment [4]. The preparation of monodisperse particles in the sub-micron range is particularly challenging because it is just between the limits of particles size by conventional emulsion polymerization and suspension polymerization. Various types of methods have been employed to prepare monodisperse particles, such as multistage swollen emulsion polymerization successive seed emulsion polymerization and suspension polymerization. However, the above-mentioned procedures are timeconsuming and often difficult to carry out. Dispersion polymerization is a simple effective method for preparing monodisperse polymer particles, which suits not only a wide variety of monomers, but also permits easy functionalization.

Recently, monodisperse polymers have found a new application in optical radiation field. The f-f transition of the rare earth ions due to the shielded by 5s, 5p full shell electron, it can be the independent luminescence center in microspheres materials [5]. Many researches have been already studied on the preparation of photoluminescence and electroluminescent rare earth complexes. In this paper, the dispersion polymerization [6] was used to fabricate rare earth functional monodisperse microspheres and explore its fluorescence properties as well.

\section{EXPERIMENTAL}

\section{A. Materials}

Methyl methacrylate (MMA, 99\%, CP) was vacuum distilled under reduced pressure before use to remove polymerization inhibitors. The purified monomer was stored at $4{ }^{\circ} \mathrm{C}$ in a nitrogen-flushed bottle for no more than one month. The initiator, 2,2-azobisisobutyronitrile (AIBN, 98\% ,CP) was recrystallized from ethanol. $\mathrm{Sm}_{2} \mathrm{O}_{3}$ was $99.9 \%$ in purity. Sm(MAA) $)_{3}$ phen pure complex was prepared, 1,10phenanthroline (phen) was used as ligand.

\section{B. Preparation of monodisperse microspheres}

PMMA microspheres were prepared by dispersion polymerization. In water/ethanol (1/8 (w/w) ) dispersion medium, based on MMA monomer, $2 \%(\mathrm{w} / \mathrm{w})$ AIBN as initiator and 1\% (w/w) PVP as stabilizer were used in the polymerization. After deaerating with nitrogen gas, the polymerization was performed at $70{ }^{\circ} \mathrm{C}$ for 10hours. And then $0.05 \%(\mathrm{w} / \mathrm{w}) \mathrm{Sm}(\mathrm{MAA})_{3}$ phen as functional monomer was added. After the addition, the mixture was kept in the same conditions for another 10hours. The resulted latex were separated by repeated centrifugation to remove the supernatant particles and washed with ethanol and doubledistilled water five times, respectively.

\section{Size-distribution of microspheres}

The size-distribution of microspheres was carried out on a laser analyzer for particles size (Master sizer2000, Malvern), which provides a Gauss curve concerning the distribution of microspheres size (percentage abundance versus diameter) using the diffractive mode of light.

\section{Images of microspheres}

Scanning electronic microscope morphology was used to characterize the morphology of microspheres, from which we can know the microspheres pictures in microcosmic size. At the same time, we can obtain the accurately diameter of the microspheres. 


\section{E. Ultraviolet and visible spectrum (UV)}

UV-spectra of microspheres were carried out on U-2010 spectrophotometer (Hitachi), equipped with software for data treatment. At ambient temperature, the solution of microspheres was putted into quartz of vessel with water as control. The change in the signal versus scanning wavelength was recorded and analyzed by the software.

\section{F. Fluorescence spectrum}

FL4500 was used to determine the fluorescence properties of the rare earth complexes. The fluorescence properties of liquid compound were characterized in DMSO.

\section{G. Thermogravimetric analysis (DTG)}

Selected Germany Netzsch STA409PC simultaneous thermal analyzer and set protective gas into nitrogen gas, heating rate to $10 \mathrm{~K} / \mathrm{min}$, the protection of the gas velocity $30 \mathrm{ml} / \mathrm{min}$, purge speed is $70 \mathrm{ml} / \mathrm{min}$, measuring temperature range for $30-900^{\circ} \mathrm{C}$, weigh auditor samples to analysis.

\section{Results AND Discussion}

The typical synthesis procedure reported above employs a stabilizer to monomer weight ratio of $1 \%$ and a monomer concentration of $15 \%(\mathrm{w} / \mathrm{w})$. We initially obtained PMMA microspheres, whose monodisperse and spherical are not very ideal. As a result, we explore the effect of changing the amount of monomer with all other variables kept constant.

\section{A. Characterization of PMMAmicrospheres}

There was no more agglomeration of the nanoparticles.

The scanning electron microscopy observation in Figure 1 shows that we have successfully synthesized the PMMA microspher0es. The microspheres have a good spherical shap and which appears uniform particle sizes. Although it is a kind of nano-material, there are no more bonds between the microspheres. And then from images below with the increase of monomer content, the particles sizes increasing, monodisperse becoming great. The monodisperse microspheres index is less than $5 \%$, and during the process of drying, the microspheres in SEM characterization appeared the self-assembling partly. Because PMMA is electron beam sensitive, resulting in a change of shape (and size) when subjected to the electron beam within the SEM. To limit the measurement error caused by deformation, images were quickly taken after exposing a region of the sample to the beam.

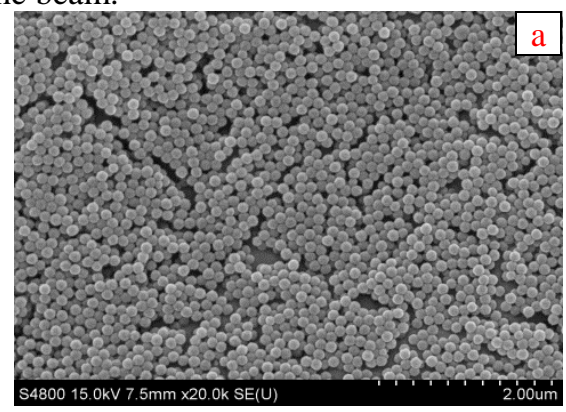

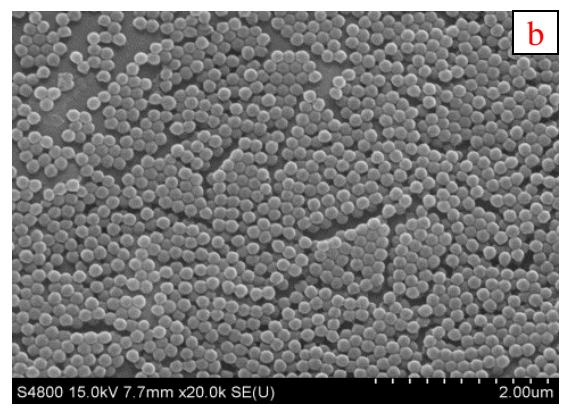

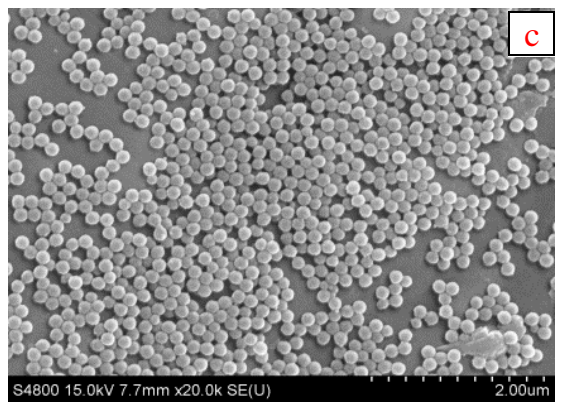

Figure 1. SEM images of different monomer content: (a, 5\% b, $10 \%$ c, 15\%)

\section{B. SEM of modified microspheres}

Figure 2 is the images of PMMA microspheres doped with rare earth. Rare earth should have been copolymerized into the polymer microspheres, and the size of microspheres becomes larger than Nano-microspheres without rare earth. And the amount of increase is about $10-20 \mathrm{~nm}$. However, the microspheres still maintained in a good spherical and monodisperse. That the addition of rare earth has not changed the mechanism of PMMA microspheres polymerization.
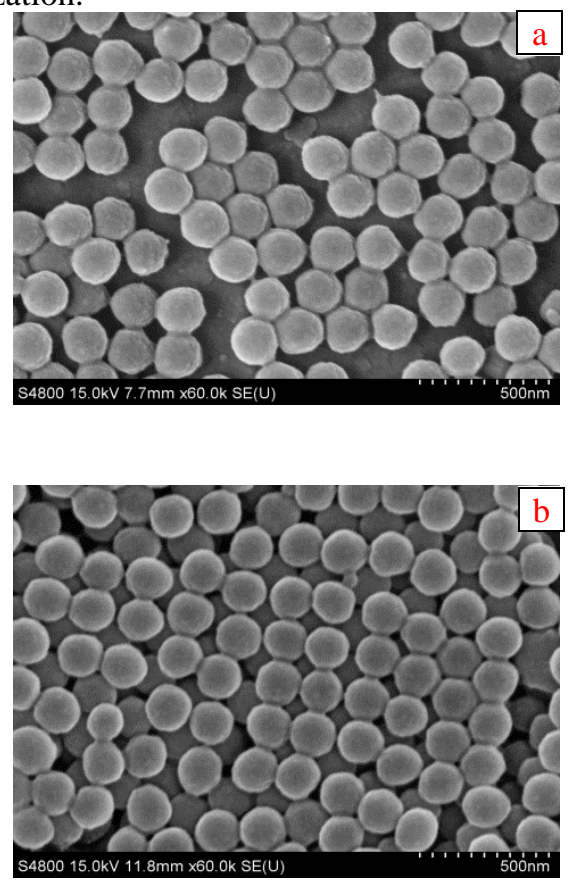

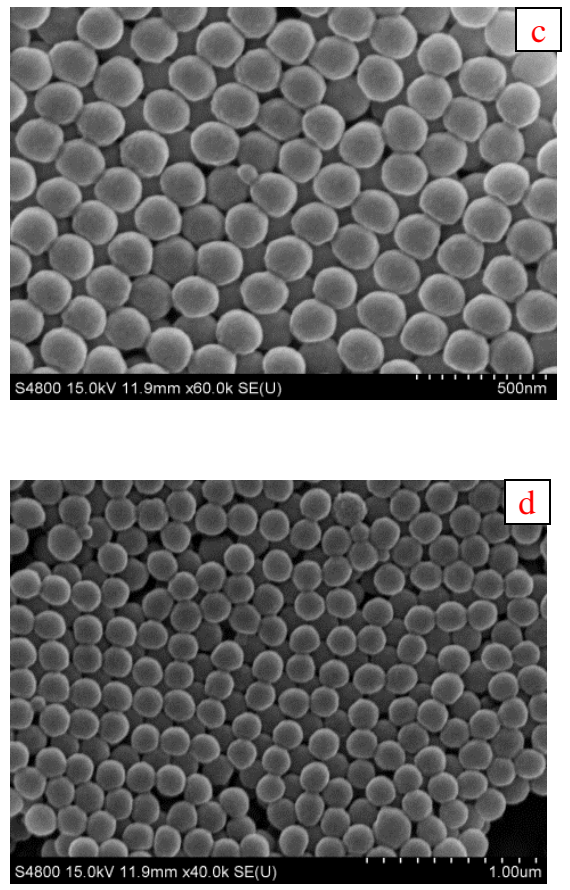

Figure 2. SEM images of PMMA microspheres doped with rare earth

(a, $5 \%$ b, $10 \%$ c, $15 \%$ d, $20 \%$ )

\section{Size-distribution of microspheres}
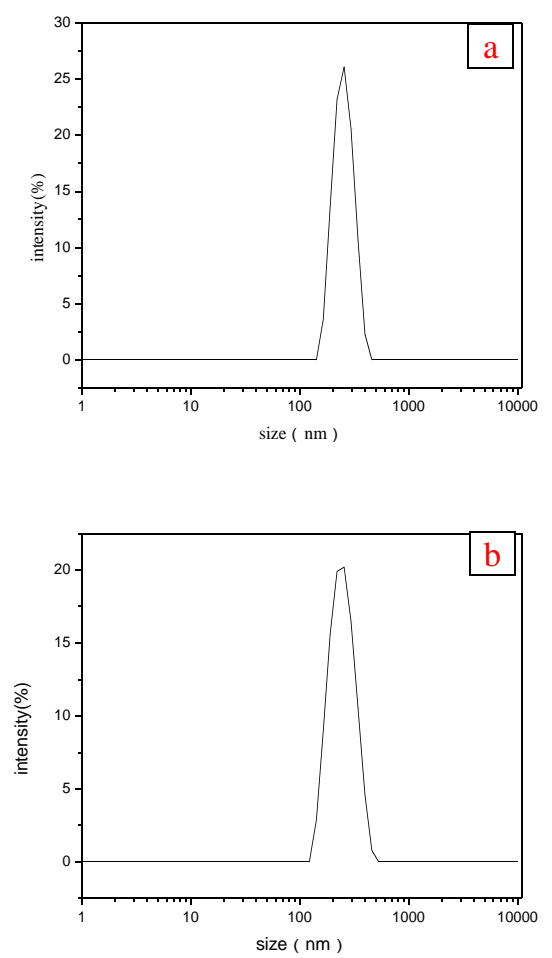

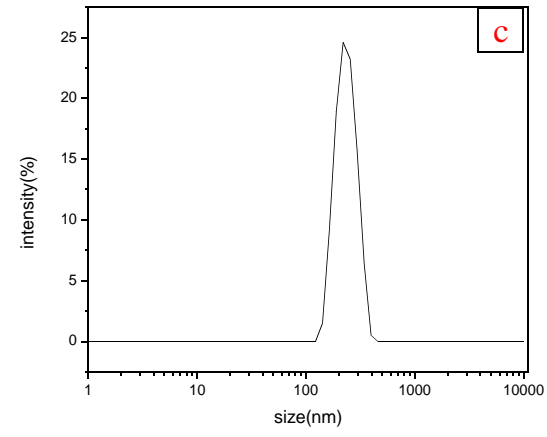

Figure 3. different content of rare earth doped in PMMAmicrospheres ( a, 5\% b, 10\% c, 15\%)

The three size distribution of microspheres with rare earth above shows that with the increase of rare earth content did not change the monodisperse copolymerization microspheres in Figure 3. By dynamic light scattering apparatus, the mean size of the microspheres increased with increasing rare earth content, and the addition are high above $10 \mathrm{~nm}$.

\section{Thermogravimetric analysis (DTG)}

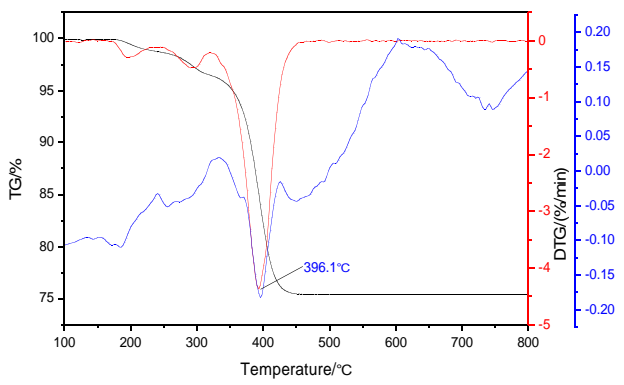

Figure 4. TG images of rare earth doped microspheres

The thermogravimetric curves of microspheres in Figure 4, indicating that the microsphere is degraded by two steps: small molecular weight components in degradation of 200$280{ }^{\circ} \mathrm{C}$; high molecular weight components in $280-420{ }^{\circ} \mathrm{C}$. 396 degrees obtained the maximum decomposition rate. 


\section{E. Ultraviolet (UV) spectrum}

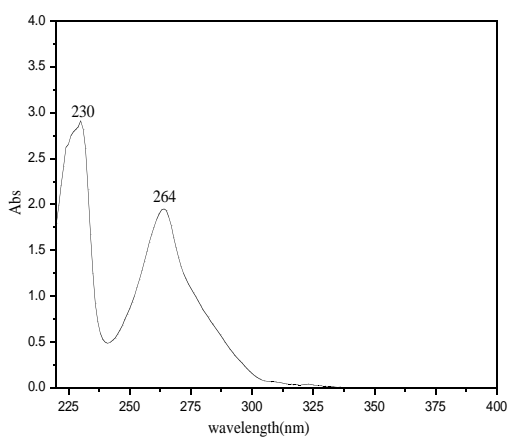

Figure 5. UV spectra of rare earth doped microspheres

Figure 5 shows the UV spectra of the modified microspheres. For intuition and clear illustration, the UV spectra of PMMA microspheres with rare earth complexes, which shows that rare earth complexes have been copolymerized into PMMAmicrospheres.

\section{F. Fluorescence spectrum}
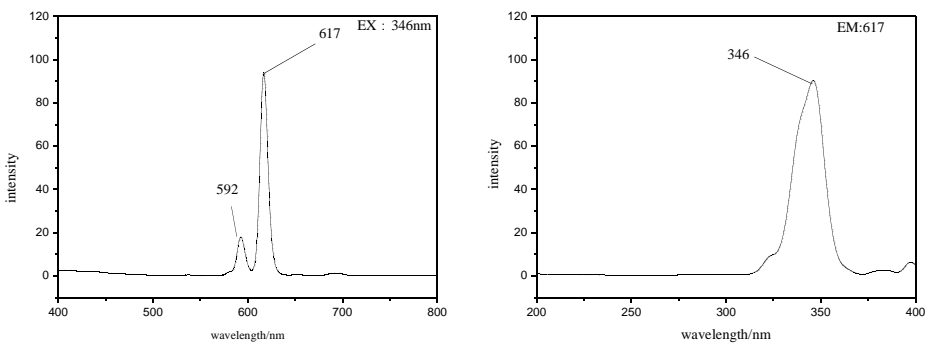

Figure 6. Emission/ Excitation spectra of rare earth doped microspheres

The typical emission spectrum of the modified microspheres is shown in Figure 6. The fluorescence lines of $\mathrm{Sm} 3+$ at around $600 \mathrm{~nm}$ were assigned to transitions from the $5 \mathrm{D}_{0}$ levels to all the multiplet levels of $7 \mathrm{~F}$. This emission spectrum was obtained under excitation at $335 \mathrm{~nm}$, corresponding to the $\mathrm{Sm}^{3+7} \mathrm{~L} 7$ levels.

\section{iv. Conclusions}

Monodisperse PMMA particles in the range of 200$400 \mathrm{~nm}$ were obtained by dispersion polymerization of MMA monomer in the water-ethanol media using polyvinyl pyrrolidone (PVP) as polymerizable stabilizer.

\section{REFERENCES}

[1] Q. Dai, and D. Zhen, "Preparation of monodisperse poly (methyl methacrylate) particles," Polymer, vol. 44, 2008, pp. 73-77.

[2] S.J. Li, and J. Hu, "use of chemically modified PMMA microspheres for enzyme immobilization,” BioSystems, vol. 77, 2004, pp. 25-32.

[3] S.M. Klein, V.N. Manoharan, D.J. Pine, F.F. Lange, "Preparation of monodisperse PMMA microspheres in nonpolar solvents by dispersion polymerization with a macromonomeric stabilizer," Colloid Polymer Sci, 2003, pp. 7-13.
[4] K.P. Lok, and C.K. Ober, "particle size control in dispersion of polystyrene,” 2004, pp. 209-212.

[5] K.M. Keville, E.I. Franses, and J.M. Caruthers, "preparation and characterization of monodisperse polymer microspheroids," Colloid and inerfare science, 1991, pp. 103-106.

[6] F.C. Schwab, and J.G. Murray (1985), Anionic dispersion polymerization of styrene. In:Culbertson BM, McGrath JE (eds) Advances in polymer synthesis, Plenum, New York. 